\title{
Recombinant Infectious Bronchitis Coronavirus Beaudette with the Spike Protein Gene of the Pathogenic M41 Strain Remains Attenuated but Induces Protective Immunity
}

\author{
Teri Hodgson, Rosa Casais, Brian Dove, $\uparrow$ Paul Britton, and Dave Cavanagh* \\ Institute for Animal Health, Division of Molecular Biology, Compton Laboratory, \\ Compton, Newbury, Berkshire, United Kingdom
}

Received 1 April 2004/Accepted 9 August 2004

\begin{abstract}
We have replaced the ectodomain of the spike (S) protein of the Beaudette strain (Beau-R; apathogenic for Gallus domesticus chickens) of avian infectious bronchitis coronavirus (IBV) with that from the pathogenic M41 strain to produce recombinant IBV BeauR-M41(S). We have previously shown that this changed the tropism of the virus in vitro (R. Casais, B. Dove, D. Cavanagh, and P. Britton, J. Virol. 77:9084-9089, 2003). Herein we have assessed the pathogenicity and immunogenicity of BeauR-M41(S). There were no consistent differences in pathogenicity between the recombinant BeauR-M41(S) and its apathogenic parent Beau-R (based on snicking, nasal discharge, wheezing, watery eyes, rales, and ciliostasis in trachea), and both replicated poorly in trachea and nose compared to M41; the $S$ protein from the pathogenic M41 had not altered the apathogenic nature of Beau-R. Both Beau-R and BeauR-M41(S) induced protection against challenge with M41 as assessed by absence of recovery of challenge virus and nasal exudate. With regard to snicking and ciliostasis, BeauRM41(S) induced greater protection (seven out of nine chicks [77\%]; assessed by ciliostasis) than Beau-R (one out of nine; 11\%) but less than M41 (100\%). The greater protection induced by BeauR-M41(S) against M41 may be related to the ectodomain of the spike protein of Beau-R differing from that of M41 by $4.1 \%$; a small number of epitopes on the $S$ protein may play a disproportionate role in the induction of immunity. The results are promising for the prospects of S-gene exchange for IBV vaccine development.
\end{abstract}

Avian Infectious bronchitis coronavirus (IBV) is in the genus Coronavirus, family Coronaviridae, order Nidovirales (21, 22, $32)$. Together with the genetically closely related turkey coronavirus $(13,26)$ and pheasant coronavirus $(14)$, they form the group 3 coronaviruses. It has a 27.6-kb single-stranded RNA genome of positive polarity associated with a nucleocapsid $(\mathrm{N})$ protein, surrounded by an envelope in which are present the large spike (S) glycoprotein, a smaller integral membrane (M) protein, and a few copies of a much smaller envelope (E) protein.

IBV is primarily a respiratory pathogen of domestic fowl, though the oviduct can be affected and some strains are nephropathogenic $(7,15)$. In young chickens it causes reduction in weight gain and a predisposition to secondary bacterial infections, which can be fatal, and also affects egg production. The problem is compounded by the existence of dozens of serotypes; cross-protection is poor $(7,18,25,27,43)$. Control is by prophylaxis using live vaccines based on several serotypes, plus inactivated vaccines in the case of breeding stock and commercial egg layers. The $\mathrm{S}$ protein (approximately 1,145 amino acid residues) is the main inducer of protective immunity $(8,10,28,29,42)$, and it is the most variable of the IBV proteins, with serotypes commonly differing by 20 to $25 \%$, and sometimes $50 \%$, of amino acids in the amino-terminal half (S1)

\footnotetext{
* Corresponding author. Mailing address: Institute for Animal Health, Compton Laboratory, Compton, Newbury, Berkshire, RG20 7NN United Kingdom. Phone: 441635 577273. Fax: 441635577263. E-mail: dave.cavanagh@bbsrc.ac.uk.

$\dagger$ Present address: Division of Microbiology, School of Biochemistry and Molecular Biology, University of Leeds, LS2 9JT United Kingdom.
}

of the $\mathrm{S}$ protein $(1,23,30,31,33,41)$. It is believed that it is the variation in $\mathrm{S}$ that is responsible for poor cross-protective immunity. It is possible that replacing the $S$ gene of a vaccinal strain with that from a heterologous strain would produce a vaccine able to induce protective immunity against the heterologous strain. A reverse genetics system enabled us to investigate this possibility $(5,6)$.

Researchers have previously assembled a full-length DNA copy of the IBV Beaudette-CK genome and cloned it in vaccinia virus, from which infectious IBV (Beau-R) was recovered (6). Recently we constructed a recombinant IBV with the genome of Beau-R, except that the ectodomain (all of $\mathrm{S}$ protein except for the transmembrane domain and carboxy-terminal cytoplasmic tail) had been replaced with corresponding sequence from the pathogenic M41 strain to produce BeauRM41(S) (5). Beaudette and M41 are both of the Massachusetts serotype, having $95 \%$ amino acid identity in S1 (3). In primary chick kidney cells, Beau-R, M41, and BeauR-M41(S) replicated equally well (5). However, whereas Beau-R replicated in Vero, BHK, and primary chicken embryo fibroblasts, M41 and BeauR-M41(S) replicated very poorly or not at all in these cell types; the M41 S-protein ectodomain resulted in alteration of the host cell range of the Beaudette strain in vitro (5).

Investigations with other coronaviruses have shown that the $\mathrm{S}$ protein is a determinant of pathogenicity; indeed, of tissue tropism in vivo. For example, the $\mathrm{S}$ protein of the group 1 porcine coronavirus, transmissible gastroenteritis coronavirus (TGEV), determines whether it has an enteric tropism or is largely limited to replication in the respiratory tract $(2,40)$. Recombinants of murine hepatitis coronavirus (MHV) containing S-protein genes of other strains of MHV replicated to 
different extents and caused differing degrees of hepatitis (35). Similarly, the MHV S protein is a determinant of neurovirulence $(34,38,39)$. It was conceivable, therefore, that exchanging the S-protein ectodomain of apathogenic Beau-R with that of pathogenic M41 would result in the recombinant virus BeauR-M41(S) being pathogenic. Clearly, this would be an undesirable outcome from a vaccine perspective, though it would be informative with regard to the role of the $\mathrm{S}$ protein in vivo.

Not only is the Beaudette strain apathogenic, a consequence of $>200$ passages in embryonated chicken eggs, but it is also considered to be poorly immunogenic (24). Consequently, it has never been used as a vaccinal strain.

There were three objectives to this study. First, to establish if the replacement of the ectodomain sequence of the S-protein gene of apathogenic Beau-R with that of pathogenic M41 would transform Beau-R into a pathogenic virus. Second, to see if this recombinant IBV, the first to be made, had the capacity to induce protective immunity against challenge with $\mathrm{M} 41$, in which case it could form the basis of a model for the future investigation of cross-protection. Third, to compare five clinical and two other criteria by which the pathogenicity of IBV might be assessed.

\section{MATERIALS AND METHODS}

Virus strains. The pathogenic M41 strain (19), which had been propagated in tracheal organ cultures (TOCs) (17), was passaged twice in embryonated chicken eggs, 11 times in primary chick kidney (CK) cells, and once more in embryonated eggs (to produce M41-CK, subsequently referred to as M41). The highly embryoadapted Beaudette strain of IBV was passaged eight times in CK cells to give Beaudette-CK (11), and a preparation was made in 10-day-old embryonated chicken eggs. The Beau-R strain was recovered from a full-length clone of Beaudette-CK (6); they are isogenic except for two nucleotide substitutions, $C^{19666 \rightarrow} U$ (serine-to-leucine substitution in the replicase gene) and $A^{27087 \rightarrow} G(a$ silent mutation in the $\mathrm{N}$ gene) (6). Beau-R was used after seven passages in $\mathrm{CK}$ cells. BeauR-M41(S) was recovered from the full-length clone after replacing the ectodomain-encoding part of the S-protein gene of Beau-R with that of M41 (5). The resulting virus, BeauR-M41(S), was passaged five times in CK cells. H120 is a commercial live IBV Massachusetts serotype vaccine strain, attenuated by approximately 120 passages in embryonated chicken eggs.

Titration of IBV. Titrations of virus infectivity were performed in TOCs prepared from specific-pathogen-free (SPF) Rhode Island Red chicken embryos (17), and the titers expressed as the $50 \%$ (median) ciliostatic doses $\left(\mathrm{CD}_{50}\right)$. TOCs were performed in glass test tubes individually, with $1 \mathrm{ml}$ of medium (17). Three or five TOCs were inoculated with virus $(0.5 \mathrm{ml})$ in serial dilutions of either tracheal suspension or ground nasal turbinate supernatant and were rotated at one revolution every $9 \mathrm{~min}$ at $37^{\circ} \mathrm{C}$ for 6 days. The TOCs were observed for ciliary activity; those exhibiting less than $5 \%$ mean activity were recorded as positive for the presence of IBV.

Growth curves in tracheal organ cultures. Groups of five TOCs in test tubes were inoculated with $0.5 \mathrm{ml}$ of medium containing $1.7 \log _{10} \mathrm{CD}_{50}$ of virus. After $1 \mathrm{~h}$ at $37^{\circ} \mathrm{C}$, the medium was removed, the TOCs were washed three times, and incubation was continued with $1 \mathrm{ml}$ of medium. At selected time points, medium from three tubes corresponding to each virus were removed and stored at $-70^{\circ} \mathrm{C}$ for subsequent titration in CK cells. RNA was extracted from the TOCs by the RNeasy method (QIAGEN) for subsequent reverse transcription-PCR (RTPCR) and sequence analysis (5) of part of the S-protein gene to confirm the identity of the virus at the end of the experiment.

Inoculation of chickens. SPF Rhode Island Red chickens were housed in positive-pressure, HEPA-filtered isolation rooms in which each group of birds was housed in a separate room. In two experiments, 8-day-old chicks were inoculated with $3.0 \log _{10} \mathrm{CD}_{50}$ of virus in a total of $0.1 \mathrm{ml}$ of $0.2 \%$ bovine serum albumen (BSA) in phosphate-buffered saline (PBS) via conjunctival (eye drop) and intranasal routes. There were 20 and 30 birds per group in experiments 1 and 2 , respectively. In the second experiment, a second (challenge) inoculation was given in the same manner 21 days after the primary inoculation. Mock-infected controls were inoculated with $0.1 \mathrm{ml}$ of $0.2 \%$ BSA in PBS.
Assessment of pathogenicity. Chicks were monitored daily for clinical signs. Snicks (a sound, somewhat analogous to a sneeze) from groups of 10 to 15 birds (two such groups per virus), were counted by three people over a 2-min period. Birds were checked individually for tracheal rales (a sound emanating from the bronchi, also detected by vibrations when holding a chick), nasal discharge, watery eye, and wheezing (dyspoena).

At necropsy, the trachea was removed for histopathological analysis (hematoxylin-eosin staining) and for assessment of ciliary activity (10). Ten 1-mm sections of each trachea were observed by lower-power microscopy. The degree of ciliostasis of each ring (the approximate percentage of lumenal surface where the cilia were no longer beating) was estimated, and the following scores were given: 0 (normal ciliary activity, corresponding to complete protection in a challenged bird), $25,50,75$, or $100 \%$ (the latter complete ciliostasis corresponding to complete absence of protection in a challenged bird).

Virus replication in chickens. For each virus, halves of three or four tracheas were cut longitudinally and the epithelia were scraped repeatedly with a scalpel blade and transferred to $1 \mathrm{ml}$ of cell culture medium (37) with 1,000 $\mathrm{U}$ of each penicillin and streptomycin per ml. Samples were stored at $-80^{\circ} \mathrm{C}$. Nasal turbinates were cut in half dorsoventrally and homogenized in medium (37) with 1,000 $\mathrm{U}$ each of penicillin and streptomycin/ml to produce a $10 \%$ suspension. The suspensions were centrifuged briefly, and the supernatants were used for titration of virus infectivity in TOCs.

Histopathology. Pieces of trachea were fixed in $10 \%$ formalin, and transverse sections were prepared and stained with hematoxylin and eosin.

Statistical analyses. Proportional data (nasal discharges, rales, watery eyes, wheezing) were analyzed by binary logistic regression, and a weighted analysis of variance (ANOVA; general linear model) was used for continuous data (snicking, ciliary activity, and virus titers). $P$ values of less than 0.05 were considered significant.

\section{RESULTS}

Beau-R with the S-protein ectodomain of pathogenic strain M41 remained apathogenic. Given that the $\mathrm{S}$ protein has been shown to be a determinant of pathogenicity with some coronaviruses, we undertook two experiments to see if the apathogenic Beau-R would be transformed into a pathogenic strain when expressing the S-protein ectodomain derived from the pathogenic M41 strain. There were 20 and 30 chicks per group in experiments 1 and 2, respectively. In both experiments chicks were inoculated with $3.0 \log _{10} \mathrm{CD}_{50}$ of infectious virus, intranasally and by eye drop, at 8 days of age, and clinical signs observed for up to 14 days. Three chicks per group per day were killed on several successive days, and their tracheae were examined for ciliary activity and infectious virus. In the second experiment infectious virus in the nose was also quantified. The commercial vaccine strain H120 was included in the first experiment.

After inoculation of chickens, infectious M41 was maximal in the trachea at 3 days after inoculation and then fell slightly over the next 2 days, after which the titer dropped markedly (Fig. 1A and B). In contrast, the levels of Beau-CK (the strain of Beaudette from which the recombinant Beau-R had been derived), Beau-R, and BeauR-M41(S) were approximately $\geq 2$ and $4 \log _{10} \mathrm{CD}_{50} / \mathrm{ml}$ lower in experiments 1 and 2, respectively. Small amounts of the H120 vaccinal strain were detected spasmodically. In the second experiment, nasal turbinates were also assayed for infectious virus; the results (data not shown) were very similar to those for tracheae. Nasal titers of M41 were 3.8, 3.9, and $2.1 \log _{10} \mathrm{CD}_{50} / \mathrm{ml}$ on days 3, 4, and 5, respectively, and they were $\leq 1.3 \log _{10} \mathrm{CD}_{50} / \mathrm{ml}$ for Beau-R and BeauR-M41(S). Thus, these two viruses had replicated poorly in the nose.

Pathogenic strains of IBV can reduce ciliary activity in the trachea by $>95 \%$ by 4 days after inoculation. This was the case in both experiments following inoculation with pathogenic M41 (Fig. 1C and D). In contrast, there were no significant 

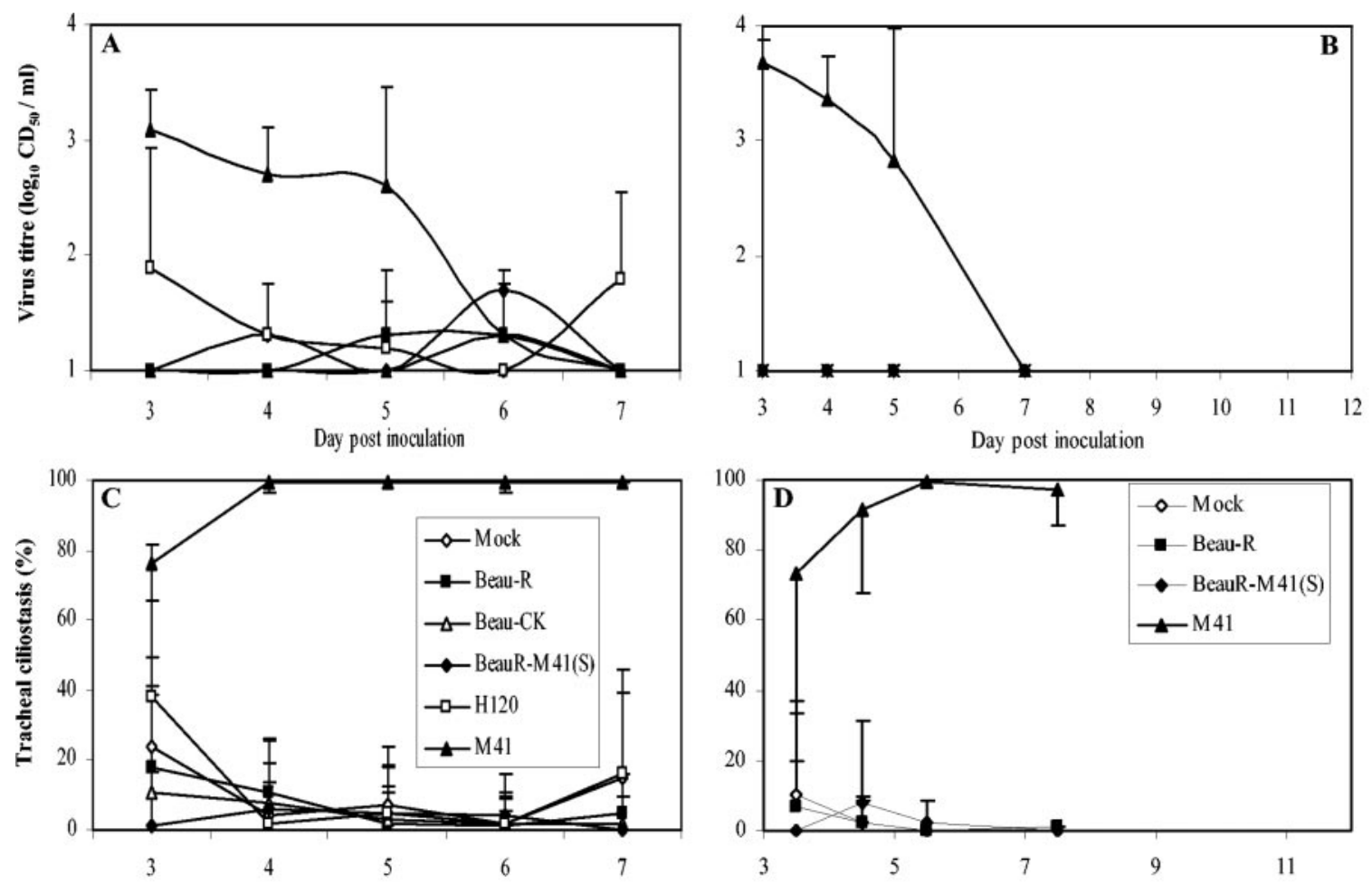

Day post inoculation
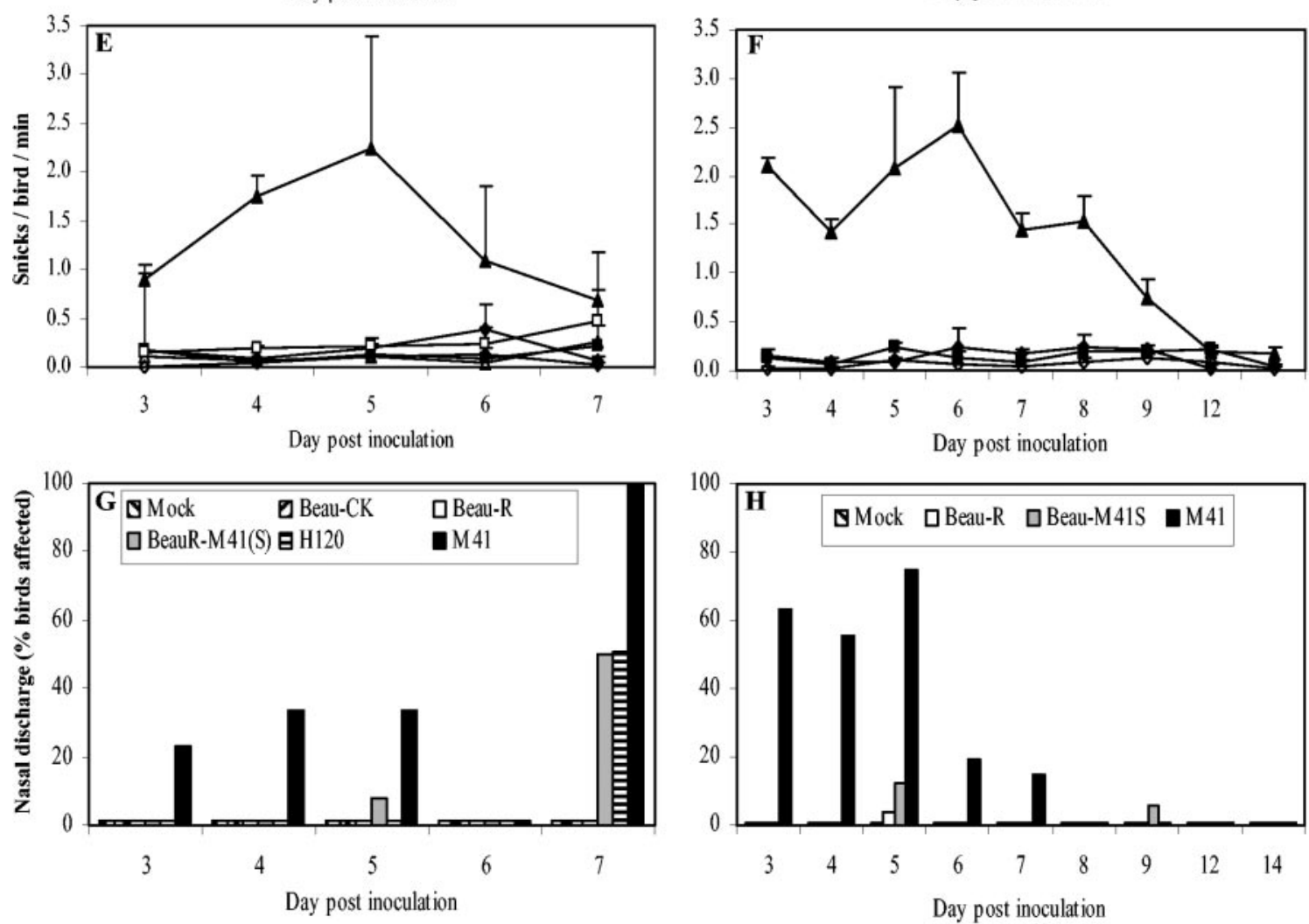

FIG. 1. Comparison of the pathogenicity of IBV strains M41 (donor of the S-protein ectodomain gene sequence), Beau-R (receiver of the S-protein ectodomain gene sequence), and BeauR-M41(S) (Beau-R with the S-protein ectodomain gene sequence of M41). Groups of 20 (experiment 1, panels A, C, E, and G) and 30 (experiment 2, panels B, D, F, and H) 8-day-old chicks were inoculated intranasally or by eye drop with $3.0 \log _{10} \mathrm{CD}_{50}$ of virus. Mock-infected chicks were inoculated with buffer only. Clinical signs were recorded from days 3 to 7 (experiment 1 ) and from days 3 to 12 (experiment 2). In experiment 1 there were two additional groups, inoculated with either Beaudette-CK (the strain from which the full-length clone of Beau-R had been derived) or H120, a commercial vaccine strain. The observations were made on individual birds, except for snicking, where birds were observed as a group. (A and B) Titer of infectious virus in tracheal scrapings, mean of three tracheas; (C and D) ciliostasis in tracheal rings, mean of three tracheas; ( $\mathrm{E}$ and F) snicking (recorded on a group basis); $(\mathrm{G}$ and $\mathrm{H})$ nasal discharge (mean of all chicks). M41 was significantly more pathogenic than the other strains $(P<0.05)$ except with respect to nasal discharge $(\mathrm{G})$. 

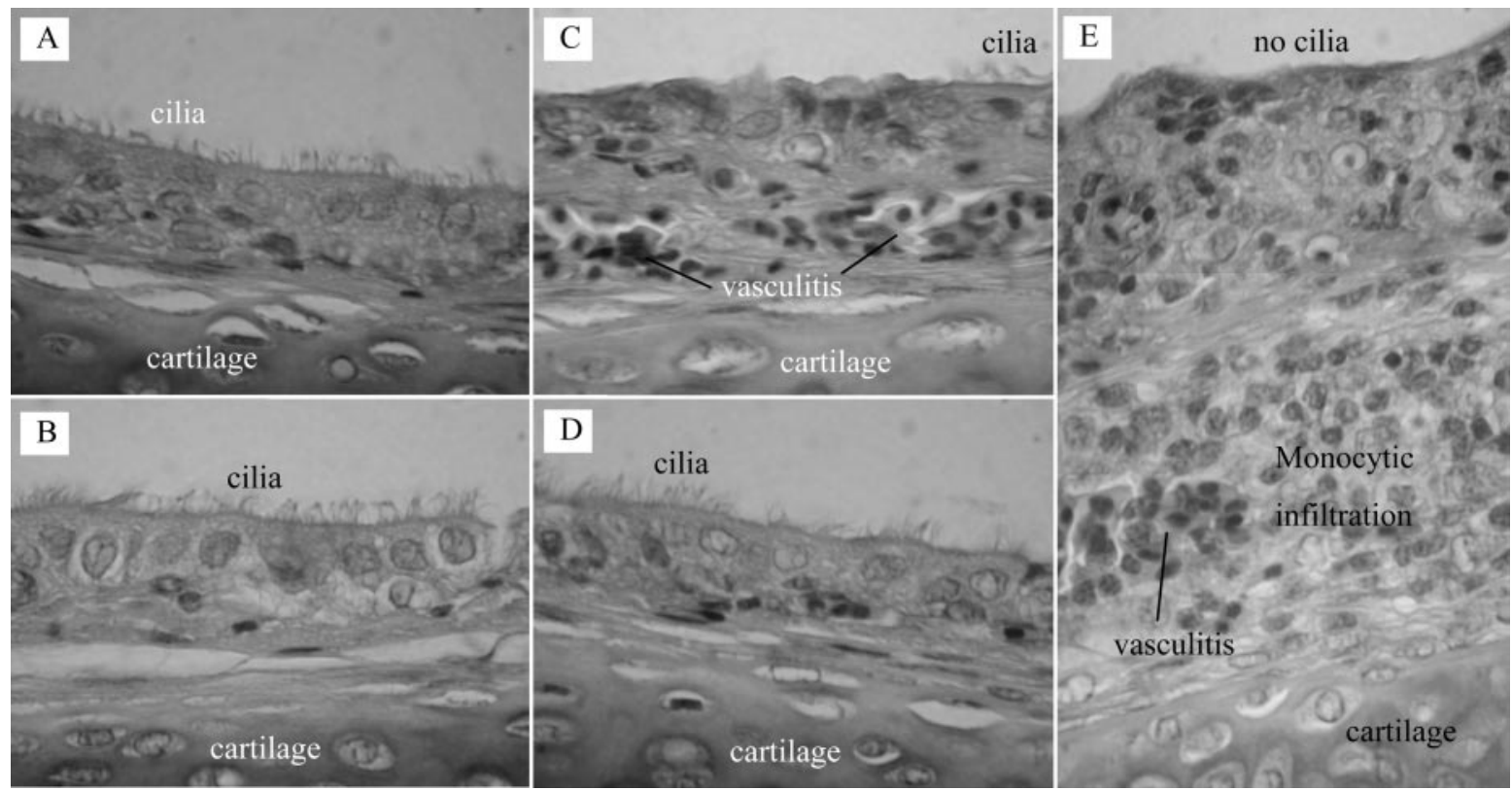

FIG. 2. Histopathology of tracheas from chickens on day 4 after inoculation. Transverse sections were stained with hematoxylin-eosin. (A) Mock infected; (B to E) infected with (B) Beau-R, (C) H120, (D) BeauR-M41(S), and (E) M41. In contrast to panels A to D, panel E shows complete deciliation and marked monocytic infiltration in the lamina propria. The H120 strain (C) caused mild deciliation and some monocytic infiltration and vascular congestion. Magnification, $\times 1,000$. (E) Composite of three photographs of the same section.

differences in ciliary activity between the mock-inoculated group and those inoculated with Beau-CK, Beau-R, BeauRM41(S), or H120. (The ciliostasis observed on the first day of trachea removal, day 3 postinoculation, in experiment 1 , was due to operator damage.)

There were no statistically significant differences in the rates of snicking (equivalent to sneezing) between the mock-inoculated chicks and those inoculated with H120, Beau-R, BeauCK, or BeauR-M41(S) (Fig. 1E and F). In contrast, the pathogenic M41 strain induced high rates of snicking, peaking at 5 days after inoculation, as expected.

Inoculation with M41 resulted in nasal discharges in 100 and $75 \%$ of the chicks in the first and second experiments, respectively (Fig. $1 \mathrm{G}$ and $\mathrm{H}$ ). In experiment 1, BeauR-M41(S) and H120 induced little or no nasal discharge on days 3 to 5, rising to $50 \%$ on day 7 after inoculation, whereas no discharge developed after inoculation with Beau-R or Beau-CK. In the second experiment, nasal discharges were induced in only 1 out of $26(4 \%)$ and 3 out of $25(12 \%)$ birds inoculated with Beau-R and BeauR-M41(S), respectively, on day 5.

Chicks inoculated with M41 exhibited rales (a sound emanating from the bronchi, also detected by vibrations when holding a chick) maximally at 58 and $43 \%$ in the first and second experiments, respectively (data not shown). Beau-R did not induce rales in either experiment, whereas BeauR-M41(S) did so in $63 \%$ of birds of experiment 1 but in none of the birds in experiment 2 . This variation, plus the very low induction of rales by M41 in a subsequent challenge experiment (reported below), indicated that rales was not a dependable criterion. The H120 vaccinal strain caused rales in $83 \%$ of chicks in experiment 1 .

M41 was the only virus to cause wheezing, being maximally $53 \%$ (day 5) and 24\% (day 6) of chickens in experiments 1 and
2, respectively (data not shown). No birds exhibited a watery eye in experiment 1 , and only birds exposed to M41 did so in experiment 2 (maximally $22 \%$ on day 4 ).

Histopathological analysis revealed that in M41-infected chickens there was, as expected, marked deciliation, desquamation, monocytic infiltration, epithelial hyperplasia, and vascular congestion (Fig. 2E). These phenomena were not observed with the mock-, Beau-R-, or BeauR-M1(S)-infected birds (Fig. 2A, B, and D). Tracheae from those birds infected with the H120 strain displayed mild deciliation, vascular congestion, monocytic infiltration, and occasional epithelial hyperplasia (Fig. 2C) but were more similar in microscopic appearance to those of the mock-infected birds than to those inoculated with M41.

In summary, although BeauR-M41(S) did induce nasal discharge and rales, this was in only one of the experiments and was at a level similar to that caused by the H120 vaccinal strain. In the other experiment, BeauR-M41(S) was as nonpathogenic as Beau-R. With respect to the more reproducible effectsrecovery of infectious virus, ciliostasis, and snicking-BeauR$\mathrm{M} 41$ (S) was as nonpathogenic as Beau-R, with both having markedly less effect than M41.

Replication in tracheal organ cultures. The recovery of very small amounts of infectious Beau-R and BeauR-M41(S) from chickens, retention of ciliary activity, and lack of snicking would have resulted if these viruses were intrinsically unable to replicate in tracheal cells. Colwell and Lukert (16) have demonstrated that the Beaudette strain does cause ciliostasis in TOCs, as we have (we titrated our stocks of Beaudette and recombinant viruses in TOCs). To investigate this further, the replication of Beau-R, BeauR-M41(S), and M41 in TOCs was quantified by titration on CK cells. All three viruses replicated in the TOCs to within $1 \log _{10}$ of each other (Fig. 3). 


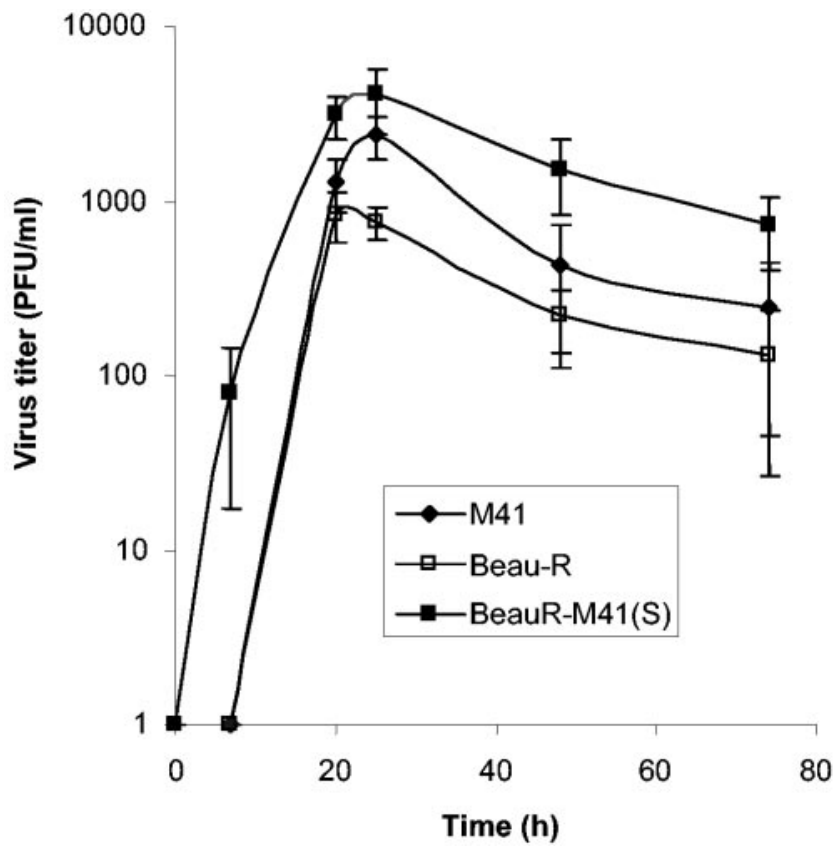

FIG. 3. Comparison of the growth curves in TOCs of IBV strain M41 (donor of the S-protein ectodomain gene sequence), Beau-R (receiver of the S-protein ectodomain gene sequence), and BeauRM41(S) (Beau-R with the S-protein ectodomain gene sequence of M41). Groups of five TOCs were inoculated with $1.7 \log _{10} C_{50}$ of virus in $0.5 \mathrm{ml}$ of medium. After $1 \mathrm{~h}$ at $37^{\circ} \mathrm{C}$ the medium was removed, the TOCs were washed three times, and incubation was continued with $1 \mathrm{ml}$ of medium. At selected time points, medium from three tubes of each virus was titrated in CK cells (37).

BeauR-M41(S)-induced protection. Experiment 2 was extended to see if any protective immunity had been induced by Beau-R and BeauR-M41(S). Three weeks after the primary inoculation, the remaining chicks (approximately 20 per group) were challenged with M41 (3.0 $\left.\log _{10} \mathrm{CD}_{50}\right)$ applied intranasally and by eye drop. Protection induced by the primary inocula was assessed by the criteria described above.

Replication of the challenge virus was assessed in three chicks at 4 days after challenge by assay of infectious virus in the trachea. Virus was detected in all three positive control birds (designated the mock:M41 group) and in none of the other groups, suggestive of the induction of protection by all three viruses (Fig. 4A).

Ciliostasis in trachea was assessed at 4, 5, and 6 days after challenge; low ciliostasis would have been indicative of a protective response. As expected, the nonchallenged birds (mock: mock group in Fig. 4B) exhibited $<5 \%$ ciliostasis, while the birds that had not received a primary inoculation but which had been challenged (mock:M41) displayed $>95 \%$ ciliostasis. Chicks that had received M41 as both primary and challenge inocula (M41:M41 in Fig. 4B) exhibited $<5 \%$ ciliostasis, demonstrating the induction of a strong protective response by the primary inoculum of this pathogenic strain, as expected. In birds that had received Beau- $\mathrm{R}$ as the primary inoculum and which had been challenged (Beau-R:M41 group in Fig. 4B), there was ciliostasis of $85 \%$ in the chickens overall. In one out of nine birds there was $45 \%$ ciliostasis, in two out of nine birds there was $80 \%$ ciliostasis, and in the remainder there was
$>95 \%$ ciliostasis. Thus, using ciliostasis as a criterion of protection, Beau-R had induced poor protection against challenge with M41. Taking $\leq 50 \%$ ciliostasis as an arbitrary criterion of protection (8), the number of birds considered protected after primary inoculation with Beau-R was $11 \%$ (one out of nine). In contrast, BeauR-M41(S) induced protection against ciliostasis in most of the birds. Thus, in the BeauR-M41(S):M41 group (Fig. 4B) there was overall ciliostasis of only $14 \%$. Seven of the nine birds had $\leq 20 \%$ ciliostasis, and five of these seven birds had $<5 \%$ ciliostasis. The other two out of nine chicks had approximately $66 \%$ ciliostasis. Therefore, taking $<50 \%$ ciliostasis as an arbitrary level for protection, the number of birds considered to be protected after primary inoculation with BeauR-M41(S) was $77 \%$ (seven out of nine) in this group of chickens.

The mock:M41 group exhibited nasal discharge for the first 4 days of the observation period, reaching maximum at $62 \%$ on day 5 after challenge (Fig. 4C). In contrast, only one bird on 1 day in the Beau-R:M41 group had a nasal exudate, while none in the BeauR-M41(S):M41 group had nasal exudates, indicative of the induction of protection by both of these viruses.

There were no rales in any groups on days 5 and 6 after challenge, except in the mock:M41 group. However, as the rales in the latter group were in only 15 and $23 \%$ of the birds, respectively, no firm deduction can be made by this criterion.

The maximum snicking rate in birds that had not received a primary inoculum but which were challenged (at 29 days of age; mock:M41 group; Fig. 4D) was less than that of the chicks that had been inoculated at 8 days of age with M41 (Fig. 1E and F), probably as a consequence of their greater age and/or larger respiratory apertures, reducing the risk of mucous causing obstructions to their airways.

Primary inoculation with M41 (M41:M41 group) had induced complete protection against challenge; snicking was as low as that in chicks that had received neither primary nor secondary challenge (mock:mock group) and as low as that in chicks that had received a primary inoculation with BeauRM41(S) but which had not been challenged [BeauR-M41(S): mock group; Fig. 4D].

The challenge virus had induced a low level of snicking by day 3 in the chicks that had received the primary inoculum of Beau-R or BeauR-M41(S). In the case of the Beau-R:M41 group, snicking continued to rise. Although it did not reach the maximum level of the chicks that had not received a primary inoculation, the differences were not significantly different. In the BeauR-M41(S):M41 group, snicking after day 3 was significantly less $(P<0.05)$ than that in the chicks that had not received a primary inoculation (mock:M41), approaching background levels. By this criterion, BeauR-M41(S) had induced a degree of protection.

Watery eyes were observed in the mock:M41 group, being maximal at $23 \%$ (day 5), and were observed in only one bird (5\% maximum, on day 3 ) in the Beau-R:M41 group and none in other groups (data not illustrated). Thus, both Beau-R and BeauR-M41(S) had induced protection against this clinical sign.

In the mock:M41 group, the maximum wheezing was only $8 \%$ (1 out of 13 ), exhibited on day 5 (data not shown). Wheezing was in 12 ( 2 out of 17 ) and $6 \%$ ( 1 out of 16 ) of the BeauR:M41 and BeauR-M41(S):M41 group chickens, respectively. Thus, neither virus appeared to have induced protection against 

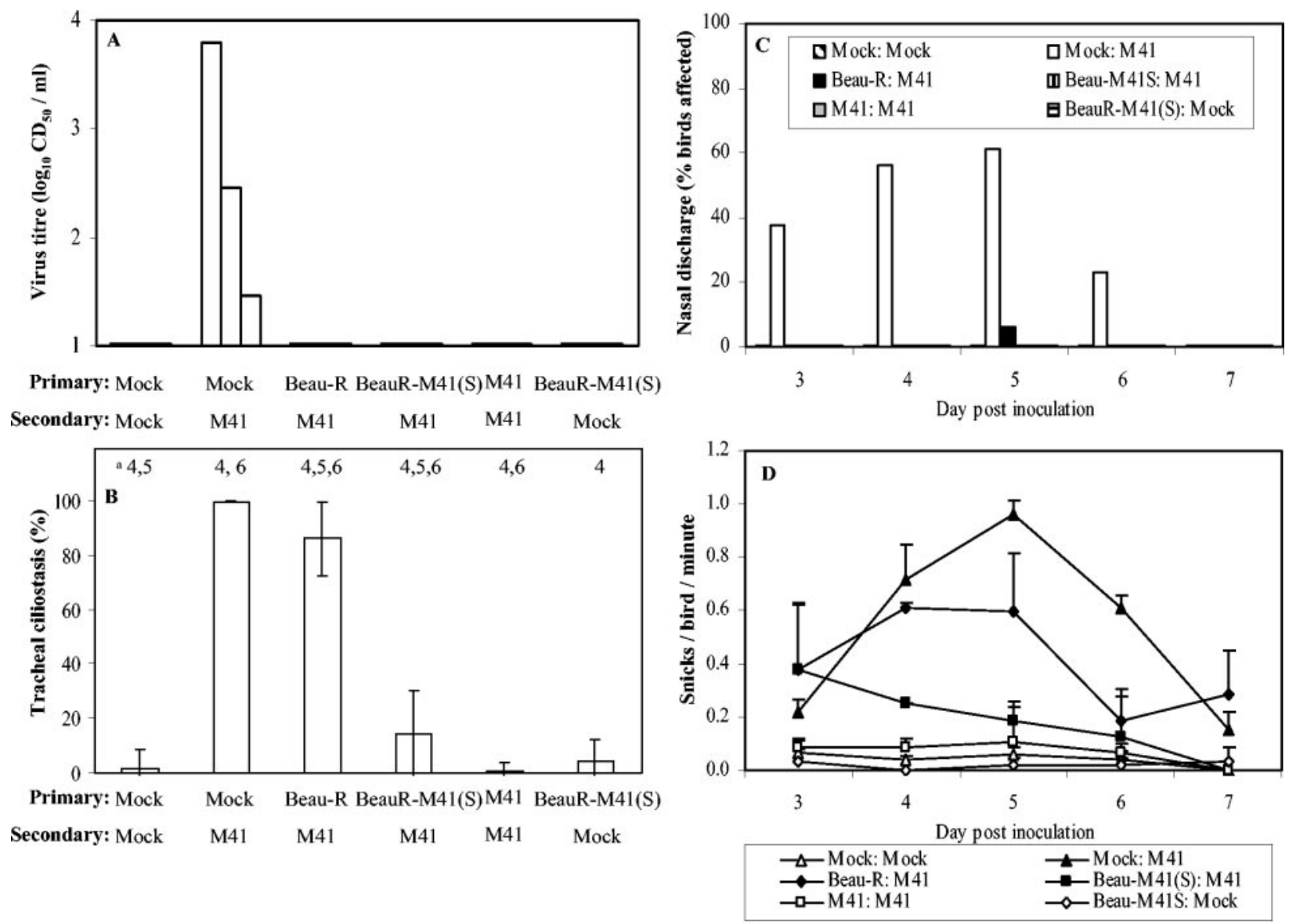

FIG. 4. Comparison of the induction of protection by IBV strains M41 (donor of the S-protein ectodomain gene sequence), Beau-R (receiver of the S-protein ectodomain gene sequence), and BeauR-M41(S) (Beau-R with the S-protein ectodomain gene sequence of M41). The birds of experiment 2 (Fig. 1B, D, F, and H) were challenged, 3 weeks after the initial inoculation, with $3.0 \log _{10} \mathrm{CD}_{50}$ of M41, applied intranasally and by eye drop. Mock-infected chicks were inoculated with buffer only. Clinical signs were recorded from days 3 to 7 . The observations were made on individual birds, except for snicking, for which birds were observed as a group. (A) Titer of infectious challenge virus recovered from the tracheae of three birds at 4 days after challenge; virus was detected in only one group, mock:M41; (B) ciliostasis in tracheal rings (mean of three tracheae collected at 4, 5, and/or 6 days after challenge, as indicated at the top; a low percentage is indicative of protection); (C) nasal discharge (mean of 15 to 18 birds examined individually); (D) snicking (15 to 18 birds examined as a group).

wheezing, although the low number of wheezing birds in the mock:M41 group diminishes the significance of this criterion.

We found it difficult to quantify histopathological changes, in part because of natural variation in noninfected chickens, e.g., with respect to depth of the submucosa. Consequently, we did not use histopathology to assess protection.

In summary, based on the six criteria of recovery of challenge virus, ciliary activity, snicking, nasal exudate, rales, and watery eyes, both Beau-R and BeauR-M41(S) had induced protective responses. With respect to two criteria (recovery of challenge virus and nasal exudate), the two apathogenic recombinants had induced protective responses as effectively as pathogenic M41. With regard to two other criteria (snicking and ciliary activity), BeauR-M41(S) had induced greater protection than Beau-R, although it was somewhat less than the protection induced by M41.

\section{DISCUSSION}

The objectives of this study were to establish if the S-protein ectodomain of a pathogenic IBV strain would confer a patho- genic phenotype on the attenuated Beaudette strain and to determine if the recombinant virus induced protective immunity. A number of parameters for quantifying pathogenicity and protective immunity were employed.

Assessment of immunity to challenge with IBV is most commonly done by removal of trachea at 4 or 5 days after challenge followed by either quantification of ciliostasis $(12,18)$ or detection of live-challenge virus $(27,36)$.

Histopathological changes have been recorded less frequently, and clinical signs were rarely measured. Winterfield et al. (43) recorded rales and snicking during experiments in which chickens vaccinated with one serotype of IBV were challenged with several heterologous serotypes. Based on the criterion of reisolation of challenge virus, cross-protection was deemed poor in some cases, whereas good cross-protection across all the serotypes was concluded when protection against rales was the criterion. In order to get a comprehensive estimation of the pathogenicity of our recombinant IBV and induction of protective immunity, we recorded five clinical signs in addition to measuring ciliostasis and virus replication. 
Snicking was the most useful of the clinical signs for defining pathogenicity, there being a numerically high difference between pathogenic and apathogenic viruses in both experiments (Fig. 1E and F). It was also a good indicator of protection (Fig. 4D). Nasal discharge was also a good indicator, though it was more variable between experiments (Fig. $1 \mathrm{G}$ and $\mathrm{H}$ ). Rales were a somewhat less useful indicator of pathogenicity, as they were less consistent than snicking. Wheezing declined as the chicks grew, reducing its value, while watery eyes was not a consistent clinical sign, with a low maximum of $22 \%$ with M41 diminishing its value.

Titration of virus from tracheae (Fig. 1A and B) and nasal turbinates gave very similar results and clearly differentiated pathogenic from apathogenic strains, as did assessment of ciliostasis. Measurement of ciliostasis was more discriminatory with regard to assessment of protective immunity (Fig. 4B) than quantification of recovered challenge virus (Fig. 4A). In conclusion, snicking, nasal discharge, tracheal ciliostasis, and challenge virus recovery were all useful parameters for assessing pathogenicity and protection.

Beaudette, like other IBVs that have been serially passaged many times in embryonated eggs (e.g., the H120 vaccine strain, 120 embryo passages, used in experiment 1), is apathogenic in chickens (24 and our data) (Fig. 1). Geilhausen et al. (24), who inoculated chickens by spray, reported that while M41 infected and destroyed the ciliated epithelial cells of the trachea, Beaudette infected only a few of them and caused little desquamation. Our histopathological examination confirmed this, as did our observation of ciliary activity (Fig. 1C and D). It is not clear why this should be, as Beaudette causes ciliostasis when inoculated onto TOCs $(4,16,44,45)$ and produced titers of virus that were similar to those of M41 and BeauR-M41(S) (Fig. 3).

BeauR-M41(S) resembled its avirulent parent Beau-R, with regard to pathogenicity, rather than the virulent donor of its S-protein gene, M41 (Fig. 1). The three viruses replicated virtually identically in CK cells (5) and similarly in TOCs (Fig. 3 ). This shows that the ciliated tracheal epithelium cells had receptors that could be utilized by both Beaudette and M41 and that the fusion of virus and cell membranes required for infection was enabled by the spike protein of both viruses. The in vivo and in vitro experiments indicate that the apathogenic nature of Beaudette is not due solely to differences between it and M41 in the S-protein ectodomain; mutations acquired during embryo passage in other genes most likely contribute to its lack of pathogenicity. The results also show that possession of an S-protein ectodomain from a virulent strain is not necessarily sufficient to overcome the attenuating mutations in other genes. In the cases of spike gene alterations of TGEV and MHV (2, 20, 34, 35, 38, 39, 40), the starting viruses were already capable of good replication in their respective hosts, i.e., the subsequent changes were essentially to tropism rather than from apathogenic to pathogenic phenotype. In contrast, IBV Beaudette had been passaged hundreds of times in embryonated eggs, resulting in an apathogenic phenotype. The chimeric S protein of BeauR-M41(S) has the cytoplasmic domain of the Beaudette $S$ protein. The cytoplasmic domain of the M41 S protein is nine amino acids shorter than that of Beaudette (3). It is unlikely that the absence of these nine residues correlates with the pathogenicity of M41, as some other virulent strains lack these nine amino acids and many other virulent strains have a cytoplasmic domain as long as that of nonvirulent Beaudette.

Our finding that Beau-R remained nonpathogenic when expressing the S protein from pathogenic M41 is promising with respect to vaccine development. IBV exists as scores of serotypes $(7,18,25,27,43)$. Cross-protection is insufficient for commercial purposes, so a number of serotypes have been used to make attenuated live vaccines. Given the major role of the $\mathrm{S}$ protein in immunity $(8,28,29,42)$, it is possible that an existing vaccinal strain could be updated-made more crossprotective-if its spike protein gene were replaced by that from a present field strain of a different serotype. Clearly, it would be disadvantageous if replacing the spike protein gene of a vaccinal strain with that from a field isolate reversed the attenuated phenotype of the vaccine. Our results indicate that this would not necessarily happen.

Gielhausen et al. (24) reported that chickens inoculated with Beaudette produced detectable virus-neutralizing (VN) antibodies, even though replication in the ciliated epithelium had been poor. The chickens were not challenged. We have shown that both Beau-R and BeauR-M41(S) stimulated protective responses.

With regard to two criteria (snicking and ciliary activity), BeauR-M41(S) had induced greater protection than Beau-R. These viruses differed only in their $\mathrm{S}$ proteins, which differed by $4.7 \%$ in the ectodomain (comprising S1 and most of S2) and by $5.0 \%$ in $\mathrm{S} 1$. It is the $\mathrm{S} 1$ subunit that induces $\mathrm{VN}$ antibodies and protective immune responses $(8,28,29,42)$.

This suggests that some of the 5.0\% (25) of S1 amino acid differences between the spike proteins of M41 and Beau-R might have contributed to epitopes that induced protective responses specifically against M41. The degree of cross-protection among IBV serotypes (serotype being defined by VN antibodies) generally diminishes as the differences between the $\mathrm{S}$ proteins increases $(7,12)$. Although IBV serotypes commonly differ by 20 to $25 \%$ in $\mathrm{S} 1(1,23,30,31,33,41)$, some serotypes differ in S1 by as little as $2 \%$ (10 amino acids) (9), suggesting that there may only be a few epitopes that induce most of the VN antibody. Vaccination with one isolate followed by challenge with two strains that differed from the vaccine strain in S1 by 2 and $4 \%$, respectively, resulted in a lower degree of protection than that derived from homologous challenge (12). Nix et al. (36) inoculated chickens with several virulent isolates that had 93 to $97 \%$ S1 amino acid identity. All were assessed as being of the same serotype, but some crossreacted in a VN test much less than others. Some of the isolates induced protection in $100 \%$ of chicks, deduced by failure to reisolate the challenge virus. However, one of the isolates induced protection in only $58 \%$ of chicks when the challenge virus was of the lower serological and sequence $(93 \%)$ identity. These reports and the result of our crossprotection experiment suggest that perhaps a small number of epitopes on the $\mathrm{S}$ protein play a disproportionate role in the induction of protection.

\section{ACKNOWLEDGMENTS}

T. Hodgson was the holder of a Research Studentship from the Biotechnology and Biological Sciences Research Council (BBSRC). The work was supported by the BBSRC, the Department of the En- 
vironment, Food and Rural Affairs (project code OD0712), and the Commission of the European Union RTD program Quality of Life and Management of Living Resources (project code QLK2-CT-199900002).

We thank Regina D'Arce, a visiting research student from the University of Campinas, Campinas, Brazil, for excellent assistance with the chicken experiments.

\section{REFERENCES}

1. Adzhar, A., R. E. Gough, D. Haydon, K. Shaw, P. Britton, and D. Cavanagh. 1997. Molecular analysis of the 793/B serotype of infectious bronchitis virus in Great Britain. Avian Pathol. 26:625-640.

2. Ballesteros, M. L., C. M. Sanchez, and L. Enjuanes. 1997. Two amino acid changes at the $\mathrm{N}$-terminus of transmissible gastroenteritis coronavirus spike protein result in the loss of enteric tropism. Virology 227:378-388.

3. Binns, M. M., M. E. G. Boursnell, F. M. Tomley, and T. D. K. Brown. 1986. Comparison of the spike precursor sequences of coronavirus IBV strains M41 and 6/82 with that of IBV Beaudette. J. Gen. Virol. 67:2825-2831.

4. Butler, M., and W. J. Ellaway. 1972. Comparative studies on the infectivity of avian respiratory viruses for eggs, cell cultures and tracheal explants. J. Comp. Pathol. 82:327-332.

5. Casais, R., B. Dove, D. Cavanagh, and P. Britton. 2003. Recombinant avian infectious bronchitis virus expressing a heterologous spike gene demonstrates that the spike protein is a determinant of cell tropism. J. Virol. 77: 9084-9089.

6. Casais, R., V. Thiel, S. G. Siddell, D. Cavanagh, and P. Britton. 2001. Reverse genetics system for the avian coronavirus infectious bronchitis virus. J. Virol. 75:12359-12369.

7. Cavanagh, D. 2003. Severe acute respiratory syndrome vaccine development: experiences of vaccination against avian infectious bronchitis virus. Avian Pathol. 32:567-582.

8. Cavanagh, D., and P. J. Davis. 1986. Coronavirus IBV: removal of spike glycopolypeptide S1 by urea abolishes infectivity and haemagglutination but not attachment to cells. J. Gen. Virol. 67:1443-1448.

9. Cavanagh, D., P. J. Davis, J. K. A. Cook, D. Li, A. Kant, and G. Koch. 1992. Location of the amino acid differences in the S1 spike glycoprotein subunit of closely related serotypes of infectious bronchitis virus. Avian Pathol. 21: $33-43$.

10. Cavanagh, D., P. J. Davis, J. H. Darbyshire, and R. W. Peters. 1986. Coronavirus IBV: virus retaining spike glycopolypeptide S2 but not S1 is unable to induce virus-neutralizing or haemagglutination-inhibiting antibody, or induce chicken tracheal protection. J. Gen. Virol. 67:1435-1442.

11. Cavanagh, D., P. J. Davis, D. J. C. Pappin, M. M. Binns, M. E. G. Boursnell, and T. D. K. Brown. 1986. Coronavirus IBV: partial amino terminal sequencing of spike polypeptide S2 identifies the sequence Arg-Arg-Phe-Arg-Arg at the cleavage site of the spike precursor propolypeptide of IBV strains Beaudette and M41. Virus Res. 4:133-143.

12. Cavanagh, D., M. M. Ellis, and J. K. A. Cook. 1997. Relationship between sequence variation in the S1 spike protein of infectious bronchitis virus and the extent of cross-protection in vivo. Avian Pathol. 26:63-74.

13. Cavanagh, D., K. Mawditt, M. Sharma, S. E. Drury, H. L. Ainsworth, P. Britton, and R. E. Gough. 2001. Detection of a coronavirus from turkey poultry in Europe genetically related to infectious bronchitis virus of chickens. Avian Pathol. 30:355-368.

14. Cavanagh, D., K. Mawditt, D. d. B. Welchman, P. Britton, and R. E. Gough. 2002. Coronaviruses from pheasants (Phasianus colchicus) are genetically closely related to coronaviruses of domestic fowl (infectious bronchitis virus) and turkeys. Avian Pathol. 31:181-193.

15. Cavanagh, D., and S. Naqi. 2003. Infectious bronchitis, p. 101-119. In Y. M. Saif, H. J. Barnes, J. R. Glisson, A. M. Fadly, L. R. McDougald, and D. E. Swayne (ed.), Diseases of poultry, vol. 11. Blackwell, Ames, Iowa.

16. Colwell, W. M., and P. D. Lukert. 1969. Effects of avian infectious bronchitis virus (IBV) on tracheal organ cultures. Avian Dis. 13:888-894.

17. Cook, J. K. A., J. H. Darbyshire, and R. W. Peters. 1976. The use of chicken tracheal organ cultures for the isolation and assay of avian infectious bronchitis virus. Arch. Virol. 50:109-118.

18. Cook, J. K. A., S. J. Orbell, M. A. Woods, and M. B. Huggins. 1999. Breadth of protection of the respiratory tract provided by different live-attenuated infectious bronchitis vaccines against challenge with infectious bronchitis viruses of heterologous serotypes. Avian Pathol. 28:477-485.

19. Darbyshire, J. H., J. G. Rowell, J. K. A. Cook, and R. W. Peters. 1979 Taxonomic studies on strains of avian infectious bronchitis virus using neutralisation tests in tracheal organ cultures. Arch. Virol. 61:227-238.

20. Das Sarma, J., L. Fu, J. C. Tsai, S. R. Weiss, and E. Lavi. 2000. Demyelination determinants map to the spike glycoprotein gene of coronavirus mouse hepatitis virus. J. Virol. 74:9206-9213.

21. Enjuanes, L., D. Brian, D. Cavanagh, K. Holmes, M. M. C. Lai, H. Laude, P. Masters, P. Rottier, S. G. Siddell, W. J. M. Spaan, F. Taguchi, and P. Talbot. 2000. Coronaviridae, p. 835-849. In M. H. V. van Regenmortel, C. M.
Fauquet, D. H. L. Bishop, E. B. Carstens, M. K. Estes, S. Lemon, J. Maniloff, M. Mayo, D. J. McGeoch, C. R. Pringle, and R. B. Wickner (ed.), Virus taxonomy. Classification and nomenclature of viruses. Academic Press, New York, N.Y.

22. Enjuanes, L., W. J. Spaan, E. J. Snijder, and D. Cavanagh. 2000. Nidovirales, p. 827-834. In M. H. V. v. Regenmortel, C. M. Fauquet, D. H. L. Bishop, E. B. Carsten, M. K. Estes, S. M. Lemon, D. J. McGeoch, J. Maniloff, M. A. Mayo, C. R. Pringle, and R. B. Wickner (ed.), Virus taxonomy. Classification and nomenclature of viruses. Academic Press, New York, N.Y.

23. Farsang, A., C. Ros, L. H. M. Renstrom, C. Baule, T. Soos, and S. Belak 2002. Molecular epizootiology of infectious bronchitis virus in Sweden indicating the involvement of a vaccine strain. Avian Pathol. 31:229-236.

24. Geilhausen, H. E., F. B. Ligon, and P. D. Lukert. 1973. The pathogenesis of virulent and avirulent avian infectious bronchitis virus. Arch. Gesampte. Virusforsch. 40:285-290.

25. Gelb, J., Jr., C. L. Keeler, Jr., W. A. K. Nix, J. K. Rosenberger, and S. S. Cloud. 1997. Antigenic and S-1 genomic characterization of the Delaware variant serotype of infectious bronchitis virus. Avian Dis. 41:661-669.

26. Guy, J. S. 2000. Turkey coronavirus is more closely related to avian infectious bronchitis virus than to mammalian coronaviruses: a review. Avian Pathol. 29:207-212.

27. Hofstad, M. S. 1981. Cross-immunity in chickens using seven isolates of infectious bronchitis virus. Avian Dis. 25:650-654.

28. Ignjatovic, J., and L. Galli. 1994. The S1 glycoprotein but not the $\mathrm{N}$ or M proteins of avian infectious bronchitis virus induces protection in vaccinated chickens. Arch. Virol. 138:117-134.

29. Johnson, M. A., C. Pooley, J. Ignjatovic, and S. G. Tyack. 2003. A recombinant fowl adenovirus expressing the $\mathrm{S} 1$ gene of infectious bronchitis virus protects against challenge with infectious bronchitis virus. Vaccine 21:2730-2736.

30. Keeler, C. L., Jr., K. L. Reed, W. A. Nix, and J. Gelb, Jr. 1998. Serotype identification of avian infectious bronchitis virus by RT-PCR of the peplomer (S-1) gene. Avian Dis. 42:275-284.

31. Kingham, B. F., C. L. Keeler, Jr., W. A. Nix, B. S. Ladman, and J. Gelb, Jr. 2000. Identification of avian infectious bronchitis virus by direct automated cycle sequencing of the S-1 gene. Avian Dis. 44:325-335.

32. Lai, M. M. C., and D. Cavanagh. 1997. The molecular biology of coronaviruses. Adv. Vir. Res. 48:1-100.

33. Lee, C. W., D. A. Hilt, and M. W. Jackwood. 2001. Identification and analysis of the Georgia 98 serotype, a new serotype of infectious bronchitis virus. Avian Dis. 45:164-172.

34. Leparc-Goffart, I., S. T. Hingley, M. M. Chua, X. Jiang, E. Lavi, and S. R. Weiss. 1997. Altered pathogenesis of a mutant of the murine coronavirus MHV-A59 is associated with a Q159L amino acid substitution in the spike protein. Virology 239:1-10.

35. Navas, S., S. H. Seo, M. M. Chua, J. Das Sarma, S. T. Hingley, E. Lavi, and S. R. Weiss. 2001. Role of the spike protein in murine coronavirus induced hepatitis: an in vivo study using targeted RNA recombination. Adv. Exp. Med. Biol. 494:139-144.

36. Nix, W. A., D. S. Troeber, B. F. Kingham, C. L. Keeler, Jr., and J. Gelb, Jr. 2000. Emergence of subtype strains of the Arkansas serotype of infectious bronchitis virus in Delmarva broiler chickens. Avian Dis. 44:568-581.

37. Pénzes, Z., K. Tibbles, K. Shaw, P. Britton, T. D. K. Brown, and D. Cavanagh. 1994. Characterization of a replicating and packaged defective RNA of avian coronavirus infectious bronchitis virus. Virology 203:286-293.

38. Phillips, J. J., M. Chua, S. H. Seo, and S. R. Weiss. 2001. Multiple regions of the murine coronavirus spike glycoprotein influence neurovirulence. J. Neurovirol. 7:421-431.

39. Phillips, J. J., M. M. Chua, E. Lavi, and S. R. Weiss. 1999. Pathogenesis of chimeric MHV4/MHV-A59 recombinant viruses: the murine coronavirus spike protein is a major determinant of neurovirulence. J. Virol. 73:7752-7760.

40. Sanchez, C. M., A. Izeta, J. M. Sanchez-Morgado, S. Alonso, I. Sola, M. Balasch, J. Plana-Duran, and L. Enjuanes. 1999. Targeted recombination demonstrates that the spike gene of transmissible gastroenteritis coronavirus is a determinant of its enteric tropism and virulence. J. Virol. 73:7607-7618.

41. Sapats, S. I., F. Ashton, P. J. Wright, and J. Ignjatovic. 1996. Sequence analysis of the S1 glycoprotein of infectious bronchitis viruses: identification of a novel genotypic group in Australia. J. Gen. Virol. 77:413-418.

42. Song, C. S., Y. J. Lee, C. W. Lee, H. W. Sung, J. H. Kim, I. P. Mo, Y. Izumiya, H. K. Jang, and T. Mikami. 1998. Induction of protective immunity in chickens vaccinated with infectious bronchitis virus S1 glycoprotein expressed by a recombinant baculovirus. J. Gen. Virol. 79:719-723.

43. Winterfield, R. W., A. M. Fadly, and F. J. Hoerr. 1976. Immunity to infectious bronchitis virus from spray vaccination with derivatives of a Holland strain. Avian Dis. 20:42-48.

44. Yachida, S., S. Aoyama, N. Takahashi, Y. Iritani, and K. Katagiri. 1979. Growth kinetics of embryo- and organ-culture adapted Beaudette strain of infectious bronchitis virus in embryonated chicken eggs. Avian Dis. 23:127-131.

45. Yachida, S., S. Aoyama, N. Takahashi, Y. Iritani, and K. Katagiri. 1978. Plastic multiwell plates to assay avian infectious bronchitis virus in organ cultures of chicken embryo trachea. J. Clin. Microbiol. 8:380-387. 